\title{
Dominant cytotoxic NK cell subset within CLPD-NK patients identifies a more aggressive NK cell proliferation
}

\author{
Gregorio Barilà ${ }^{1,2}$, Antonella Teramo ${ }^{1,2}$, Giulia Calabretto ${ }^{1,2}$, Chiara Ercolin ${ }^{1,2}$, Elisa Boscaro ${ }^{1}$, Valentina Trimarco ${ }^{1,2}$, \\ Samuela Carraro ${ }^{1}$, Matteo Leoncin ${ }^{1,2}$, Cristina Vicenzetto ${ }^{1,2}$, Anna Cabrelle ${ }^{2}$, Monica Facco ${ }^{1,2}$, Francesco Piazza ${ }^{1,2}$, \\ Gianpietro Semenzato ${ }^{1,2}$ and Renato Zambello ${ }^{1,2}$
}

Natural killer (NK) cells represent a class of innate lymphocytes with large granular morphology and cytotoxic functions, characterized by the CD3-/CD16+/CD56 + phenotype. According to CD56 expression, two major NK cell subsets can be recognized, CD $56^{\text {high }} / \mathrm{CD} 16^{\text {dim/neg }}$ NK cells with the ability to release cytokines and CD $56^{\text {dim }} /$ CD $16^{\text {high }}$ NK cells displaying cytotoxic ability toward virus infected or neoplastic cells ${ }^{1}$. NK cells are traditionally considered part of innate immunity but evidence has been recently provided that a distinct NK cell subset may respond to specific antigens like adaptive immune cells ${ }^{2}$. These NK cells, also known as "NK memory", are induced by the chronic stimulation of viral infections or by cytokines (IL-12, IL-15 and IL-18) and are included in the CD56 ${ }^{\mathrm{dim}} / \mathrm{CD} 16^{\text {high }} \mathrm{NK}$ cells subgroup equipped with $\mathrm{CD} 57$ but lacking $\mathrm{CD}^{2} 2 \mathrm{~L}^{2,3}$. Chronic lymphoproliferative disorder of NK cells (CLPD-NK) is a provisional entity, recognized by the 2016 WHO classification, characterized by chronic expansion of at least 500/ $\mathrm{mm}^{3} \mathrm{NK}$ cells with restricted killer immunoglobulin-like receptor (KIR) pattern, whose assessment is of crucial relevance due to the lack of $\mathrm{T}$-cell receptor rearrangement in NK cells ${ }^{4,5}$. CLPD-NK has generally an indolent course, most patients being asymptomatic and the main feature of the disease being represented by the development of neutropenia ${ }^{4}$. Only few data are available on the pathogenesis of this indolent disorder but a constitutive activation of anti-apoptotic signaling pathways is likely to play

\footnotetext{
Correspondence: Gianpietro Semenzato (g.semenzato@unipd.it) or Renato Zambello (r.zambello@unipd.it)

${ }^{1}$ Department of Medicine, Hematology and Clinical Immunology Section, Padua University School of Medicine, Padua, Italy

${ }^{2}$ Venetian Institute of Molecular Medicine (VIMM), Padua, Italy
}

a relevant role ${ }^{5}$. The discovery in 2012 of somatic STAT3 mutations, in about $40 \%$ of T-cell large granular lymphocyte leukemia (T-LGLL) and in about $30 \%$ of CLPDNK, focused the attention on a constitutive activation of Janus Kinase-Signal Transducers and Activators of Transcription (JAK-STAT) pathway in the development of this disorder ${ }^{6,7}$. In addition, somatic $S T A T 5 b$ mutations were recently recognized in rare aggressive variants of LGLL and indolent CD4+ T-LGLL ${ }^{8}$.

This rare disorder remains an extremely heterogeneous disease, some patients being completely asymptomatic, whereas others require specific treatment. For this reason, using flow cytometry and regardless of KIR expression, the aim of this study was to identify different biological and clinical CLPD-NK patients' subsets. By flow analysis, NK cells of 25 patients affected by CLPD-NK were analyzed for CD16 and CD56 expression (Supplementary Methods), recognizing two major NK cell subsets, that is, patients with CD56 ${ }^{\mathrm{dim}} / \mathrm{CD} 16^{\mathrm{dim}}$ NK cells $(4 / 25,16 \%)$ and patients with CD56 ${ }^{\text {neg/dim }} / C D 16^{\text {high }}$ NK cells $(21 / 25,84 \%)$ (Figs. 1a-c). All patients were evaluated for clinical and hematological characteristics. Median age was 62 years (range 42-79) with no significant difference between $\mathrm{CD} 56^{\mathrm{dim}} / \mathrm{CD} 16^{\mathrm{dim}}$ and $\mathrm{CD} 56^{\text {neg/dim }} / \mathrm{CD} 16^{\text {high }}$ subgroups. As expected, neutropenia (absolute neutrophil count $(\mathrm{ANC})<1500 / \mathrm{mm}^{3}$ ) was the most relevant feature, detected in 10 out of 25 patients (40\%), with 5 patients (20\%) presenting severe neutropenia $\left(\mathrm{ANC}<500 / \mathrm{mm}^{3}\right.$ ). Anemia and thrombocytopenia have been detected only in a minority of patients $(2 / 25$ and $3 / 25$, respectively) and were generally mild (Table 1 ). In terms of clinical presentation, almost all symptomatic patients were included in the $\mathrm{CD} 56^{\text {neg/dim }} / \mathrm{CD} 16^{\text {high }}$ subgroup with 8 out of 21 


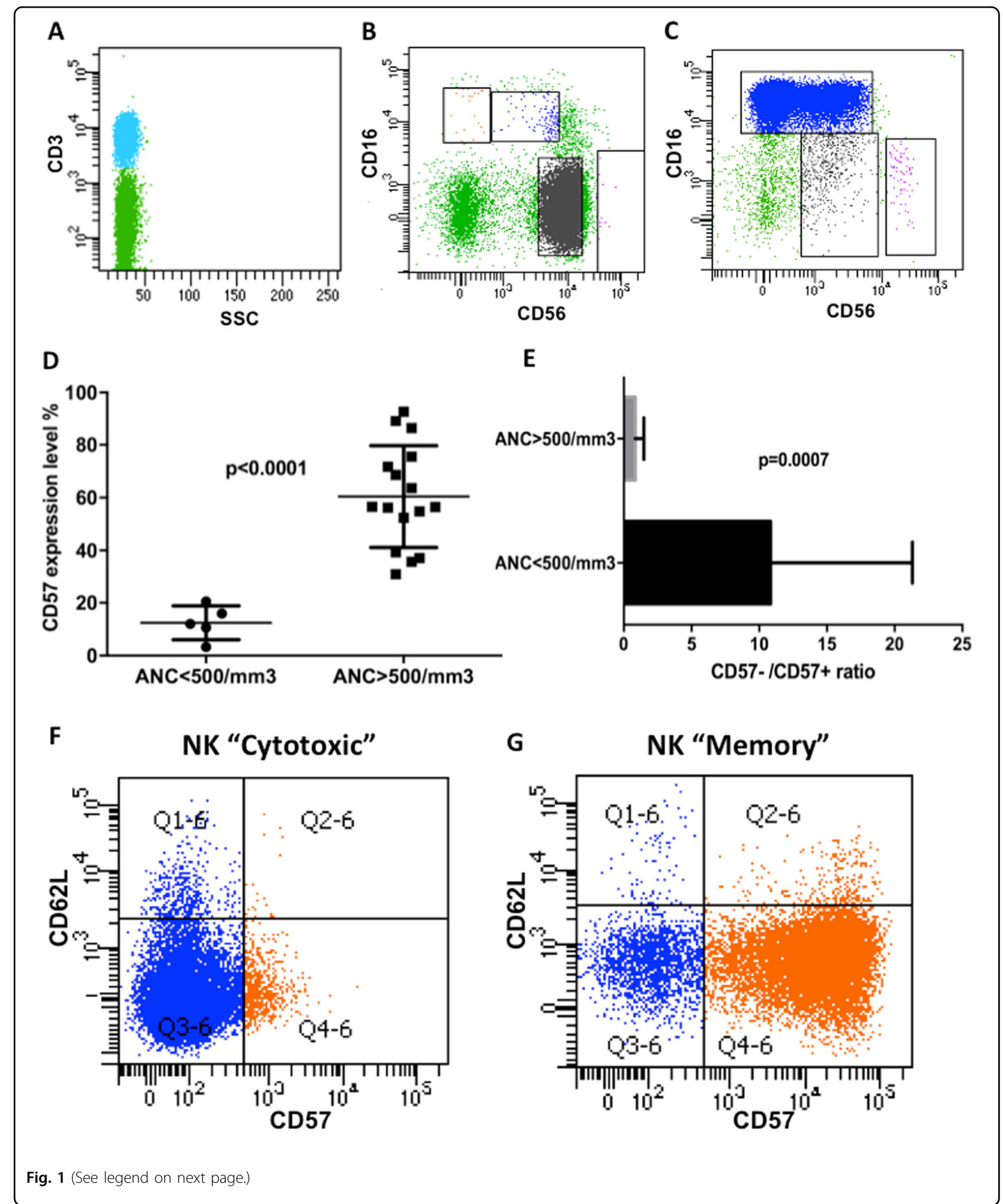

(38\%) patients presenting neutropenia and 5 out 21 (24\%) severe neutropenia. Only three patients required treatment (low-dose cyclophosphamide in two cases and methotrexate in one) during the natural history of the disease, all belonging to $\mathrm{CD} 56^{\text {neg/dim }} / \mathrm{CD} 16^{\text {high }}$ subset.

Considering the clinical heterogeneity of $\mathrm{CD} 56^{\text {neg/dim}} /$ CD16 ${ }^{\text {high }}$ subset, we analyzed the expression of CD57 on 
Fig. 1 Immunophenotypic features of CLPD-NK patients and their correlation with neutrophil count. a-c Immunophenotypic analysis of NK cells. CD3+ lymphocytes were excluded from the analysis (panel a, green). CD3- lymphocytes (panel a, blue) were studied for CD56 and CD16 relative expression identifying CD56 $6^{\text {high }} / C D 16^{\text {dim/neg }}$ NK cells (pink), CD56 dim/CD16 $\mathrm{dim}$ NK cells (gray) and CD56 dim/neg/CD16 high NK cells (blue). As a consequence, patients were classified according to the predominant NK cell subset, of notice CD56 ${ }^{\mathrm{dim}} / \mathrm{CD}^{\mathrm{dim}}$ NK cell subgroup (panel $\mathbf{b}$ ) and CD56 ${ }^{\text {dim/neg }} /$ CD16 ${ }^{\text {high }}$ NK cell subgroup (panel c). d, e Dot plot indicating differential CD57 expression (\%) in CD56 dim/neg/CD16 high $s u b g r o u p$ between patients who experienced severe neutropenia (ANC $<500 / \mathrm{mm}^{3}$ ) and patients who did not $\left(\right.$ ANC $\left.>500 / \mathrm{mm}^{3}\right)$. As represented, patients with ANC $<500 / \mathrm{mm}^{3}$ presented CD57\% expression significantly lower $(p<0.0001)$ toward patients with ANC $>500 / \mathrm{mm}^{3}$ (panel d). As a consequence, patients who experienced severe neutropenia presented a CD57-/CD57+ mean ratio significantly higher towards patients who did not ( $p=0.0007$; panel e). f, g Immunophenotypic analysis for CD57 and CD62L among CD56 dim/neg/CD16 $6^{\text {high }}$ NK cells (blue). CD57 expression (orange) discriminates two patient's subgroups characterized by CD57 negativity (panel $\mathbf{f}$ ) and positivity (panel $\mathbf{g}$ ) respectively, which we identify as "Cytotoxic" and "Memory" NK subgroups. As previously demonstrated, no significant CD62L expression was found, confirming that both subsets are constituted by terminal differentiated NK cells

Table 1 Clinical features of CLPD-NK patients

\begin{tabular}{|c|c|c|c|}
\hline & $\begin{array}{l}\text { CD56 }{ }^{\mathrm{dim}} / \\
\text { CD16 } \\
(n=4)\end{array}$ & $\begin{array}{l}\text { CD } 56^{\text {dim/neg/ }} \\
\text { CD16 } \\
+ \text { NK } \\
\text { “CD57 } \\
\text { “Memory” ( } n= \\
\text { 16) }\end{array}$ & $\begin{array}{l}\text { CD56 }{ }^{\text {dim/neg/ }} \\
\text { CD16 } \\
\text { NK "Cytotoxic } / C D 57- \\
(n=5)\end{array}$ \\
\hline CD94/NKG2A & 4/4 (100\%) & 13/16 (81\%) & $5 / 5(100 \%)$ \\
\hline CD94/NKG2C & $1 / 4(25 \%)$ & $4 / 16(25 \%)$ & $0 / 5$ \\
\hline CD158a & $1 / 4(25 \%)$ & $0 / 16$ & $0 / 5$ \\
\hline CD158b & $0 / 4$ & $8 / 16(50 \%)$ & $0 / 5$ \\
\hline CD158e & $0 / 4$ & $3 / 16(19 \%)$ & $0 / 5$ \\
\hline Neutropenia & $2 / 4(50 \%)$ & $3 / 16(19 \%)$ & $5 / 5(100 \%)$ \\
\hline Severe neutropenia & $0 / 4$ & $0 / 16$ & $5 / 5(100 \%)$ \\
\hline Treatment & $0 / 4$ & $0 / 16$ & $3 / 5(60 \%)$ \\
\hline Anemia & $0 / 4$ & $0 / 16$ & $2 / 5(40 \%)$ \\
\hline Thrombocytopenia & $0 / 4$ & $2 / 16(13 \%)$ & $1 / 5(20 \%)$ \\
\hline STAT3 mutation & $0 / 4$ & $0 / 16$ & $2 / 5(40 \%)$ \\
\hline STAT5b mutation & $0 / 4$ & $0 / 16$ & $0 / 5$ \\
\hline
\end{tabular}

cells of these patients identifying a $51 \pm 13.5 \%$ mean expression positivity. Of notice, all five patients who experienced severe neutropenia and all symptomatic patients requiring treatment presented a significantly lower CD57 mean expression toward other CD56 ${ }^{\text {neg/dim/ }}$ CD16 ${ }^{\text {high }}$ patients $(12.48 \% \pm 2.87$ vs $60.43 \% \pm 4.38, p<$ 0.0001, Fig. 1d). As a consequence CD57-/CD57+ ratio was significantly higher in symptomatic patients as compared with remnant $\mathrm{CD} 56^{\text {neg/dim }} / \mathrm{CD} 16^{\text {high }}$ patients $(10.84 \pm 5.24$ vs $0.84 \pm 0.33, p=0.0007$, Fig. 1e). Taken these data together, through immunophenotype, three major NK cell subgroups of patients can be identified. CD16 levels discriminated between CD56 ${ }^{\mathrm{dim}} / \mathrm{CD} 16^{\mathrm{dim}}$ subgroup and $\mathrm{CD} 56^{\text {neg/dim}} / \mathrm{CD} 16^{\text {high }}$ subgroup. Among the latest, CD57 expression identified patients who experienced severe neutropenia characterized by a "Cytotoxic" phenotype with CD57 negativity from less symptomatic patients characterized by CD57 expression, resembling the "NK Memory" phenotype (Figs. 1f, g, respectively). All the three subgroups displayed high CD94 expression, whereas only CD $56^{\mathrm{dim}} / \mathrm{CD} 16^{\mathrm{dim}} \mathrm{NK}$ cells expressed discrete amount of CD62L. No statistically significant differences in CD94-NKG2A/C and KIR expression were found among the three subgroups although CD94/NKG2C phenotype and KIR restriction for CD158b and CD158e were almost exclusively distinct features of NK "Memory" subgroup (8/16 and 3/16, respectively), whereas $\mathrm{CD} 56^{\mathrm{dim}} / \mathrm{CD} 16^{\mathrm{dim}}$ and NK "Cytotoxic" subgroups displayed CD94-NKG2A phenotype and presented a skewed KIR pattern characterized by lack of KIR expression ( $3 / 4$ and 5/5, respectively). All these features are reported in Table 1 .

STAT3 exon 21 mutations analysis and STAT5b exons 16 to 18 analysis were performed in our cohort of patients (Supplementary Methods). As previously reported $^{9}$, the frequency of STAT3 mutated patients was lower with respect to T-LGLL with only two (8\%) mutated patients found. These latters were characterized by the "Cytotoxic" NK immunophenotypic signature without dominant KIR expression and NKG2A pattern. In addition, all mutated patients presented severe neutropenia and required treatment during the natural history of the disease. None $S T A T 5 b$ mutated patient was found in our cohort of CLPD-NK patients (Table 1).

With the aim of identifying biological features matching with clinical characteristics, we focused on the recognition of a specific NK cell immunophenotypic signature that eventually allows a biological and clinical classification of this rare disorder. We found that, independently from KIR expression, patients characterized by CD56 ${ }^{\text {neg/ }}$ $\mathrm{dim} / \mathrm{CD} 16^{\text {high }} / \mathrm{CD} 57$ - cytotoxic NK cell expansion represent a different phenotypic subgroup characterized by symptomatic disease and by the presence of STAT3 mutation, suggesting a more aggressive NK cell proliferation. These findings agree with the article of Morice et al. $^{10}$ in which CLPD-NK patients were classified upon CD56 expression; CD56 ${ }^{\text {neg }}$ NK cell patients were 
characterized by CD16 expression, presence of cytopenia and treatment requirement. However, this analysis alone may not be enough considering that also among CD56 ${ }^{\text {neg/ }}$ dim/CD16 high patients, a different clinical behavior can be recognized. We found that patients who experience severe neutropenia and eventually require treatment belong to the CD $56^{\text {neg/dim }} / \mathrm{CD} 16^{\text {high }} /$ CD 57 - NK subset, thus conferring a prognostic role to CD57. As a further confirmation, the specific $\mathrm{CD} 56^{\text {neg } / \mathrm{dim}} / \mathrm{CD} 16^{\text {high }} / \mathrm{CD} 57-$ immunophenotypic signature is also partially associated to a specific biological hallmark of LGL leukemia, which is the presence of STAT3 mutation. As we recently demonstrated in T-LGLL ${ }^{11}$, also in this large cohort of CLPD-NK patients, immunophenotypical analysis can identify patients with symptomatic disease and might represent a suitable surrogate of STAT3 mutation sequencing. WHO 2016 classification confirmed CPLDNK as a provisional entity, emphasizing the high heterogeneity of the disease $e^{4,7,12}$. The frequency of STAT3 mutated patients found in our cohort is slightly lower as compared with what has been reported in previous studies by Jerez and Rajala ${ }^{7,12}$. This feature can be explained by a higher frequency of symptomatic and treated patients in their study populations, possibly due to enrichment in NK "Cytotoxic" patients, as herein defined. Unfortunately, in the above articles ${ }^{7,12}$, the frequency of CD57 NK cells was not reported.

Clonality assessment for NK cell neoplasms still represents an Achilles heel due to the lack of a clonotypic structure on these cells. Besides, only a small fraction of CLPD-NK reveals STAT3 mutation, the majority of patients displaying wild-type STAT3. For this reason, other biological features were assessed to prove the clonal proliferation. Historically, a skewed pattern of KIR expression was considered as a reliable surrogate of clonality to characterize pathological NK cell expansion $^{13,14}$. On the contrary, Bàrcena and colleagues ${ }^{9}$, using HUMARA assay to distinguish monoclonal $v s$ polyclonal proliferation, recognized a surrogate for NK clonality in CD $94^{\text {high }} /$ HLA-DR + signature. This approach was able to identify a concordance between the presence of STAT3 mutation and monoclonal NK cell proliferation but, surprisingly, not significant differences were found between monoclonal and polyclonal patients in terms of clinical features (specifically the presence of cytopenia), suggesting that a percentage of truly CLPD-NK symptomatic patients were missed by this classification. In addition, CD94 expression represents a specific marker of mature NK cells that is not suitable for distinction between polyclonal and monoclonal NK cell proliferations.

The etiology of LGLL is still unknown but several authors hypothesized that chronic antigenic stimulation can trigger an initial LGL proliferation that subsequently carries on as a consequence of persistent cytokine stimulation or gain of somatic mutation (i.e., STAT3 mutation $)^{5}$. The evidence that some patients are characterized by NK cells expansion with memory-like phenotype, may support this hypothesis. In fact, several studies highlighted that viral stimulation by cytomegalovirus can trigger a CD57+/NKG2C NK cell expansion with memory properties and, in some cases, this process is stimulated by cytokines, like IL-12, IL-15 and IL-18, which have been proven to play a pathogenetic role in LGLL $^{15}$.

In conclusion, through NK cell subsets flow analysis, discrete subtypes of CLPD-NK can be identified. At variance with KIR expression, which does not correlate with clinical features, patients characterized by CD $56^{\text {neg/dim/ }}$ CD16 ${ }^{\text {high }} / \mathrm{CD} 57$ - cytotoxic NK cells expansion represent a unique phenotypic subgroup characterized by more symptomatic disease and the presence of STAT3 mutation, suggesting a more aggressive proliferation of NK cells.

\section{Acknowledgements}

The authors would like to thank Associazione Italiana per la Ricerca sul Cancro (AIRC, IG-15286), Cariparo, Cariverona e Ministero dell'Università e della Ricerca Scientifica e Tecnologica (MURST).

\section{Authors' contributions}

G.B. designed the research, analyzed data and wrote the manuscript. A.T., G.C., C.E. and C.V. performed mutations analysis. E.B., S.C., A.C. and V.T. performed flow cytometer studies. M.L. provided patient's samples and patient's data. M.F. and F.P. contributed to analyze data. G.S. provided funding, participated to the analysis of data and critically reviewed and edited the manuscript. R.Z. designed the study, analyzed data, wrote the manuscript and supervised the study.

\section{Author details}

${ }^{1}$ Department of Medicine, Hematology and Clinical Immunology Section, Padua University School of Medicine, Padua, Italy. ${ }^{2}$ Venetian Institute of Molecular Medicine (VIMM), Padua, Italy

Conflict of interest

The authors declare that they have no conflict of interest.

\section{Publisher's note}

Springer Nature remains neutral with regard to jurisdictional claims in published maps and institutional affiliations.

Supplementary Information accompanies this paper at (https://doi.org/ 10.1038/s41408-018-0088-1).

Received: 9 January 2018 Revised: 5 March 2018 Accepted: 27 April 2018 Published online: 05 June 2018

\section{References}

1. Campbell, K. S. \& Hasegawa, J. Natural killer cell biology: an update and future directions. J. Allergy Clin. Immunol. 132, 536-544 (2013).

2. Min-Oo, G., Kamimura, Y., Hendricks, D. W., Nabekura, T. \& Lanier, L. L. Natural killer cells: walking three paths down memory lane. Trends Immunol. 34, 251-258 (2013)

3. Cerwenka, A. \& Lanier, L. L. Natural killer cell memory in infection, inflammation and cancer. Nat. Rev. Immunol. 16, 112-123 (2016). 
4. Semenzato, G., Marino, F. \& Zambello, R. State of the art in natural killer cell malignancies. Int. J. Lab. Hematol. 34, 117-128 (2012).

5. Lamy, T., Moignet, A. \& Loughran, T. P. Jr. LGL leukemia: from pathogenesis to treatment. Blood 129, 1082-1094 (2017).

6. Koskela, H. L. et al. Somatic STAT3 mutations in large granular lymphocytic leukemia. N. Engl. J. Med. 366, 1905-1913 (2012).

7. Jerez, A., Clemente, M. J., Makishima, H., Koskela, H. \& Leblanc, F. Peng Ng K. et al. STAT3 mutations unify the pathogenesis of chronic lymphoproliferative disorders of NK cells and T-cell large granular lymphocyte leukemia. Blood 120, 3048-3057 (2012).

8. Andersson, E. I. et al. High incidence of activating STAT5B mutations in CD4positive T-cell large granular lymphocyte leukemia. Blood 128, 2465-2468 (2016).

9. Barcena, P. et al. Phenotypic profile of expanded NK cells in chronic lymphoproliferative disorders: a surrogate marker for NK-cell clonality. Oncotarget 6, 42938-42951 (2015).
10. Morice, W. G. et al. Chronic lymphoproliferative disorder of natural killer cells: a distinct entity with subtypes correlating with normal natural killer cell subsets. Leukemia 24, 881-884 (2010).

11. Teramo, A. et al. STAT3 mutation impacts biological and clinical features of TLGL leukemia. Oncotarget 8, 61876-61889 (2017).

12. Rajala, H. L. et al. The analysis of clonal diversity and therapy responses using STAT3 mutations as a molecular marker in large granular lymphocytic leukemia. Haematologica 100, 91-99 (2015).

13. Semenzato, G. et al. The lymphoproliferative disease of granular lymphocytes: updated criteria for diagnosis. Blood 89, 256-260 (1997).

14. Zambello, R. et al. Expression and function of KIR and natural cytotoxicity receptors in NK-type lymphoproliferative diseases of granular lymphocytes. Blood 102, 1797-1805 (2003).

15. Romee, R. et al. Cytokine activation induces human memory-like NK cells. Blood 120, 4751-4760 (2012). 